\title{
Effect of the "Search AV" feature on left ventricular longitudinal deformation and ProBNP levels in patients with implanted dual- chamber pacemakers
}

\section{Adem Yılmaz ${ }^{1 *}$ \\ Mustafa Serdar Yılmazer2, \\ Feyza Kurt ${ }^{3}$}

'Kocaeli Gebze Fatih State Hospital, Department of Cardılogy, Gebze, Turkey ${ }^{2}$ Maltepe University Medical School Hospital, Department of Cardiology, Maltepe, Turkey

${ }^{3}$ Yalova State Hospital, Department of Cardiology, Yalova, Turkey

\section{RECEIVED:}

August 29, 2019

ACCEPTED:

September 16, 2019

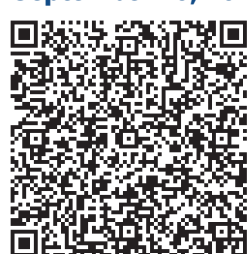

KEYWORDS: search AV, strain, speckle tracking echocardiography, Pro-BNP, dual-chamber pacemaker CITATION: Cardiol Croat. 2019;14(9-10):241. | https://doi.org/10.15836/ccar2019.241

*ADDRESS FOR CORRESPONDENCE: Adem Yılmaz, İdealtepe Mahallesi Rıfkı Tongsir Caddesi No:59/9, 34841 Maltepe/İstanbul, Turkey. / Phone: +905383270241 / E-mail: admylmz@yahoo.com

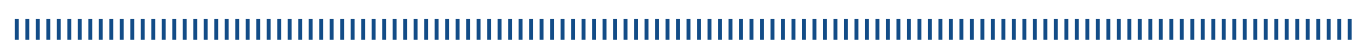

Introduction: Dual-chamber pacemaker implantation in patients with high grade AV block is a lifesaving intervention. Unfortunately, one of the most important drawbacks is its ventricular stimulation and the resultant LV systolic dysfunction due to left bundle brunch block. In recent years, in order to avoid these drawbacks and to potentialize patients' own intrinsic conduction, novel algorithms have been developed by multiple pacemaker manufacturers. "Search AV" is one of the algorithms. ${ }^{1-3}$ This study's objective is to evaluate whether LV longitudinal deformation (assessed with automated function imaging-AFI) will improve after engagement of the Search AV function. Secondary objective was comparison of serum ProBNP values levels.

Patients and Methods: It is a cross-over design study where patients remained on solely pacemaker stimulation for the first 30 days. During the second month, Search AV was engaged, and the abovementioned parameters were evaluated. At zero-point, basic pacemaker and echocardiographic parameter were measured. After 30 days, patients are switched to the "Search AV" group. After 4 weeks, the second time battery control, Speckle Tracking Echocardiography (STE) based AFI with LV longitudinal strain analysis was performed and ProBNP were measured. Echopac were analyzed with the program again.

Results: In subgroup analysis, when the cut off value for RV pacing rate was considered to be $\% 40$, in the group of ventricular pacing rate $\% 40$ and below, the decrement of ProBNP was found to be more significant by comparing $\% 40$ and higher pacing rate group $(\mathrm{p}=0.001)$. The decrement of AFI values at the end of the 2 nd month were not statistically significant $(p=0.189)$. However, when the cut off value for RV pacing rate was considered to be $\% 30$ the AFI value which demonstrates the improvement of LV function showed significant increasement $(\mathrm{p}=0.031)$ likewise statistically significant decrement of ProBNP values ( $\mathrm{p}=0.027)$.

Conclusion: Search AV is one these algorithms which reduces ventricular artificial stimulation with compromising patients' lifes. When adjusting these algorithms, target the RV pacing rate should be below $\% 30$, not $\% 40$ as mentioned in the previously published papers. Indeed, further long-term prospective studies with homogenous patients are needed to prove this argument.

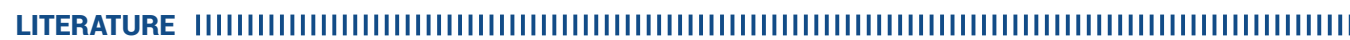

1. Tantengco MVT, Thomas RL, Karpawich PP. Left ventricular dysfunction after long-term right ventricular apical pacing in the young. J Am Coll Cardiol. 2001 Jun 15;37(8):2093-100. https://doi.org/10.1016/S0735-1097(01)01302-X

2. Gillis AM, Pürerfellner H, Israel CW, Sunthorn H, Kacet S, Anelli-Monti M, et al; Medtronic Enrhythm Clinical Study Investigators. Reducing unnecessary right ventricular pacing with the managed ventricular pacing mode in patients with sinus node disease and AV block. Pacing Clin Electrophysiol. 2006 Jul;29(7):697-705. https://doi.org/10.1111/j.1540-8159.2006.00422.x

3. Kerr CR, Connolly SJ, Abdollah H, Roberts RS, Gent M, Yusuf S, et al. Canadian Trial of Physiological Pacing: Effects of physiological pacing during long-term follow-up. Circulation. 2004 Jan 27;109(3):357-62. https://doi.org/10.1161/01.CIR.0000109490.72104.EE 